\title{
Physical and Optical Characterization of Multifunctional 3-D Self-Assembled Photonic Crystals Containing Embedded Features
}

\author{
P.V. Braun*
}

* Department of Materials Science and Engineering, Frederick Seitz Materials Research Laboratory, and Beckman Institute for Advanced Science and Technology, Urbana, IL 61801

The unifying goal of our research program is the development of self- and directed-organization as a general paradigm for the formation of highly functional nanomaterials with complex, threedimensionally embedded architectures. Specific applications where this paradigm will result in advanced materials include three-dimensional photonic crystal based chemical and biological sensors, self-assembled waveguides, and nanoparticle assemblies. Characterization of these structures requires the combination of traditional hard materials microscopy techniques, and the microscopy approaches common for biological samples. Interfacing both these approaches allows for a much more detailed picture of the self-assembled structures than would be possible through traditional routes only. We use combinations of FIB, TEM, SEM, confocal and 2-photon microscopy, and tissue fixation routes to prepare and analyze our samples.

As one example of a complex multifunctional structure, we have self-assembled 3-D photonic crystal based $\mathrm{pH}$ and glucose sensors (Figure 1) [1-2]. The sensor is created by infilling the interstitial space of a colloidal crystal with a hydrogel functionalized to respond to a specific chemical compound, followed by removal of the colloidal crystal via etching. Because of the periodic structure of the colloidal crystal template, the hydrogel is also templated with a periodic structure. The periodicity is on the order of the wavelength of light, and thus, if the structure swells or contracts, the color of the light diffracted by the hydrogel changes. The $\mathrm{pH}$ sensor has a sensitivity of $\sim 0.01 \mathrm{pH}$ units, and the glucose sensor can detect physiological glucose levels in buffers similar to blood serum.

Another example of complex self-assembled multifunctional structures we have formed and characterized are 3-D photonic band gap materials containing defined embedded structures, which may serve as laser cavities and waveguides [3]. Through a multiphoton polymerization process, we write essentially arbitrary 3-D polymer features within self-assembled colloidal crystals (Figure 2). The resulting structures have been characterized through optical spectroscopy, as well as SEM and TEM using FIB to prepare the samples. Through multiphoton polymerization, we can write 3-D voxels as small as $200 \mathrm{~nm}^{3}$ within a self-assembled matrix. Initial optical studies of these features are now underway.

References

[1] Y.-J. Lee and P. V. Braun, Adv. Mater. 15 (2003) 563.

[2] Y.-J Lee, S. A. Pruzinsky and P. V. Braun, Langmuir 20 (2004) 3096.

[3] W. Lee, S. A. Pruzinsky, and P. V. Braun, Adv. Mater. 14 (2002) 274.

[4] This work supported in part by the ARO MURI grant DAAD19-03-1-0227, the NSF grant DMR 00-71645, and the DOE grant DEFG02-91ER45439. This work was carried out in part in the UIUC CMM, UIUC, which is partially supported by the DOE under grant DEFG02-91-ER45439. 
(a)

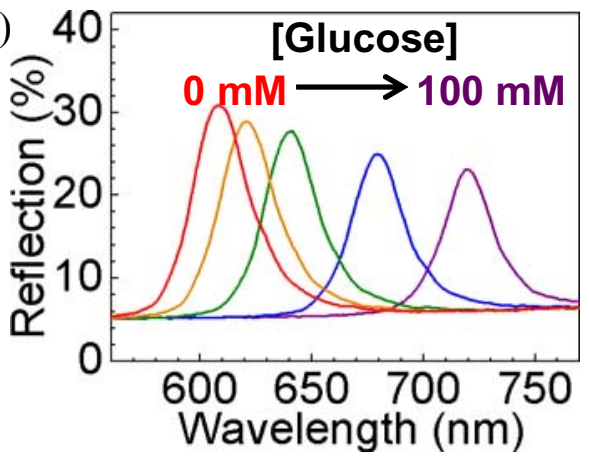

(c)

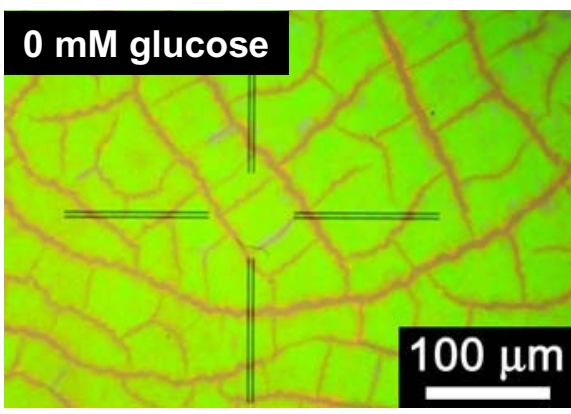

(b)

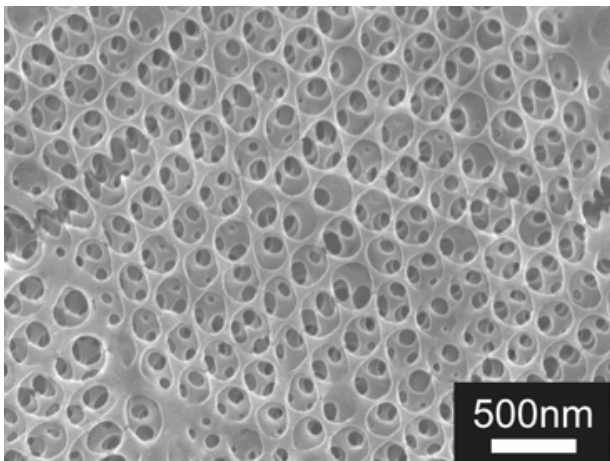

\section{$1 \mathrm{mM}$ glucose}

Fig.1. (a) optical reflectivity of a mesoporous hydrogel created by templating with a self-organized polystyrene colloidal crystal. This hydrogel is functionalized with boronic acid groups, making it sensitive to glucose at physiological ionic strength. (b) SEM micrograph of the templated hydrogel structure after removal of the colloidal crystal template. (c) color, in reflection, of the periodic hydrogel structure at $0 \mathrm{mM}$ and $1 \mathrm{mM}$ glucose.

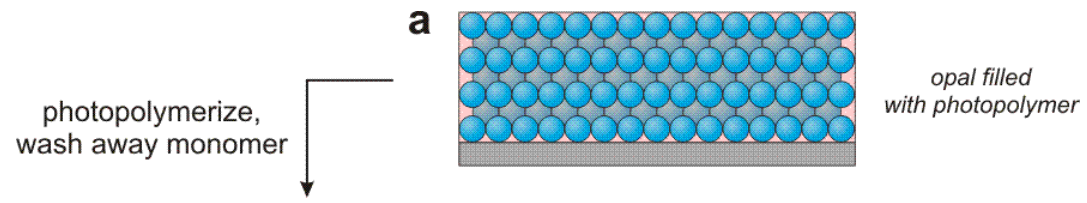

b

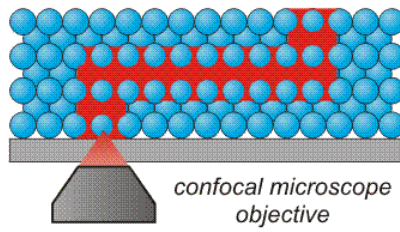

C

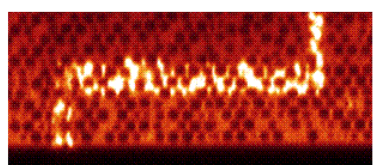

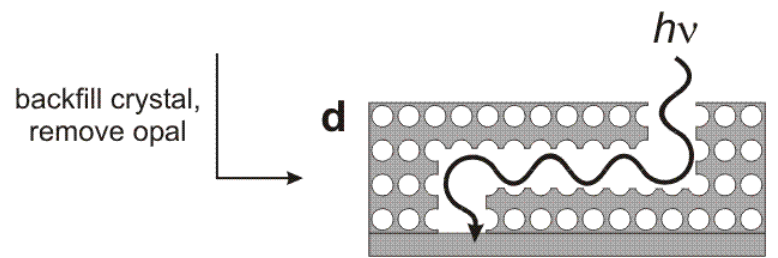

Fig.2. Schematic process for direct writing of waveguide structures in three-dimensional photonic bandgap materials. 1c is a confocal microscope image of a polymer line embedded in a 3-D silica based colloidal crystal. The dark spots are the colloidal particles (diameter $=1.6 \mu \mathrm{m}$ ), the bright line is the written feature, and dark red is the interstitial space between the particles. We have completed $\mathrm{a}-\mathrm{c}$, and are progressing towards step $\mathrm{d}$. 